\title{
RINGS WITH DUAL CONTINUOUS RIGHT IDEALS
}

\author{
SAAD MOHAMED \\ (Received 10 August 1981) \\ Communicated by R. Lidl
}

\begin{abstract}
In this paper the structure of rings with dual continuous right ideals is discussed. The main result is the following: If $R$ is a ring with (Jacobson) radical nil, and all of its finitely generated right ideals are dual continuous, then $R \simeq\left(\begin{array}{c}S \\ 0\end{array}\right)$ where $S$ is a finite direct sum of local rings each of which has its radical square zero, or is a right valuation ring, $T$ is semiprimary right semihereditary ring, and $M$ is an $(S, T)$-bimodule such that all of its finitely generated $T$-submodules are projective. A partial converse of this result is obtained: any matrix ring of the above type with $M=0$ has all of its finitely generated right ideals dual continuous.
\end{abstract}

1980 Mathematics subject classification (Amer. Math. Soc.): 16 A 10, 16 A 50, 16 A 51.

\section{Introduction}

Mohamed and Singh (1977) introduced the concept of dual continuous modules (for short d-continuous) modules as follows: A module $M$ is called d-continuous if it satisfies the following conditions: (I) for every submodule $A$ of $M$ there exists a decomposition $M=M_{1} \oplus M_{2}$ such that $M_{1} \subset A$ and $M_{2} \cap A$ is small in $M$ and (II) every epimorphism from $M$ onto a summand of $M$ splits. Any quasi-projective module over a perfect ring is d-continuous but not conversely. Over arbitrary rings the relation between dual continuity and quasi-projectivity is less close. However d-continuous modules still possess many properties which are analogous to that of quasi-projective modules. The study of d-continuous modules was motivated to generalize a decomposition theorem for quasi-projective modules over perfect rings given by Koehler (1971). A decomposition theorem for d-continuous modules over arbitrary rings was obtained by Mohamed and Singh (1977) and was later improved by Mohamed and Müller (1979) as follows:

c. Copyright Australian Mathematical Society 1982 
THEOREM. $A$ d-continuous module $M$ has a decomposition, unique up to isomorphism, $M=\Sigma_{i \in I} \oplus A_{i} \oplus N$ where each $A_{i}$ is a local module and $N=\operatorname{Rad} N$.

It follows by the above theorem that a d-continuous module with small radical is a direct sum of local modules. In particular a finitely generated $d$-continuous module is a finite direct sum of local modules.

Jain and Singh (1975) generalized the concept of hereditary rings; they called a ring $R$ right qp-ring if every right ideal of $R$ is quasi-projective. Making an effective use of Koehler's decomposition theorem, they studied perfect qp-rings. Then Goel and Jain (1976) studied semiperfect qp-rings with nil radical. Having obtained the above decomposition theorem for d-continuous modules, here we discuss rings with d-continuous right ideals.

DEFINITION. A ring $R$ in which every right ideal (resp. finitely generated right ideal) is d-continuous is called a right dc-ring (resp. right dcf-ring).

In the present work we study the structure of dc-rings and dcf-rings with nil radical. The structure of arbitrary dc-rings is still open.

All rings considered have unities and all modules are unital right modules. Rad $M$ and Soc $M$ will denote the Jacobson radical and socle of a module $M$ respectively. For any ring $R, \operatorname{Rad} R_{R}$ will be denoted by $J(R)$ or simply $J$. A module $M$ is local if $\operatorname{Rad} M$ is a maximal submodule. A ring $R$ is local if $R_{R}$ is a local module, that is $R / J$ is a division ring. For the definitions and basic properties of semiperfect and semiprimary rings, we refer to Faith (1976). If $X$ is a subset of a ring $R$, then $r(X)$ (resp. $l(X)$ ) will denote the right (resp. left) annihilator of $X$ in $R$. For any ring $R$, Soc $R_{R} \subset l(J)$ and if $R$ is local, then Soc $R_{R}=l(J)$. For definition and basic properties of quasi-projective modules we refer to Miyashita (1966) or Wu and Jans (1967).

\section{Some general results}

The following results about d-continuous modules are given in Mohamed and Singh (1977) and are listed here for easy reference.

THEOREM 2.1. A ring $R$ is (semi) perfect if and only if every ( finitely generated) quasi-projective $R$-module is d-continuous.

COROllaRY 2.2. A ring $R$ is semiperfect if and only if $R_{R}$ is d-continuous. 
LeMma 2.3. Let $A$ and $B$ be submodules of a d-continuous module $M$ such that $M=A+B$. Then there exist submodules $A_{0}$ and $B_{0}$ such that $A_{0} \subset A, B_{0} \subset B$ and $M=A_{0} \oplus B_{0}$.

LeMmA 2.4. Let $A$ and $B$ be summands of a d-continuous module $M$. Then any exact sequence $A \stackrel{f}{\rightarrow} B \rightarrow 0$ splits. If in addition $A$ is indecomposable and $B \neq 0$, then $f$ is an isomorphism.

LEMMA 2.5. If $M \times M$ is d-continuous, then $M$ is quasi-projective.

Proposition 2.6. Let $M$ be any module and $A, B$ be two small submodules of $M$ such that $M / A \oplus M / B$ is d-continuous, then $M / A \simeq M / B$.

Next we prove

Lemma 2.7. Let $M=A+B$ be a $d$-continuous module. If $A$ and $B$ are indecomposable and noncomparable, then $A \cap B=0$.

Proof. By Lemma 2.3, $M=A_{0} \oplus B_{0}$ where $A_{0} \subset A$ and $B_{0} \subset B$. Since $A$ is indecomposable $A_{0}=0$ or $A_{0}=A$. However $A_{0}=0$ implies

$$
A \subset M=B_{0} \subset B,
$$

a contradiction. Hence $A_{0}=A$. Similarly $B_{0}=B$. Hence $A \cap B=0$.

The following is well known.

LEMMA 2.8. If $R$ is a right valuation ring with $J$ nil, then any right ideal of $R$ is two-sided.

\section{Main results}

We first note that any dc-ring (or dcf-ring) is semiperfect by Corollary 2.2. This fact will be used without any further reference.

THEOREM 3.1. The following are equivalent for a ring $R$ with $J$ nil:

(i) $R$ is a right dcf-ring such that $e$ Re is a division ring for every indecomposable idempotent $e$.

(ii) $R$ is a semiprimary right semihereditary ring. 
Proof. Assume (i). Let $A$ be a right ideal of $R$ such that $A_{R}$ is local. (Such a right ideal will be called local right ideal). As $R$ is semiperfect, there exists an indecomposable idempotent $e$ of $R$ with an $R$-epimorphism $f: e R \rightarrow A$. By Lemma 2.7 either $e R \subset A$ or $A \subset e R$ or $e R \cap A=0$. If $e R \subset A$, then $e R=A$ since $A$ is indecomposable. Let $A \subset e R$. Then $f(e)=e x e \in e R e$. Since exe is a unit in $e R e$, we get $A=e R$. It remains to discuss the case when $e R \cap A=0$. Since $e R \oplus A$ is d-continuous, $A \simeq e R$ by Lemma 2.4. This all shows that $A$ is projective. Now let $B$ be a finitely generated right ideal of $R$. By the decomposition theorem of d-continuous modules, $B$ is a finite direct sum of local right ideals. Hence $B$ is projective, and $R$ is right semihereditary.

Let $R=e_{1} R \oplus \cdots \oplus e_{n} R$, for some orthogonal indecomposable idempotents $e_{i}$. We have shown that any local right ideal of $R$ is isomorphic to some $e_{i} R$, $i=1, \ldots, n$. Now, let $C$ and $D$ be distinct local right ideals of $R$ such that $C \simeq D$. We claim that $C$ and $D$ are not comparable. On the contrary, assume that $C \subset D$. Then $C$ is small in $D$. Let $D \simeq e_{i} R$. This yields a nonzero $R$-endomorphism $\phi$ of $e_{i} R$ with $\phi\left(e_{i} R\right) \subset e_{i} J$. Consequently $e_{i} J e_{i} \neq 0$, a contradiction. This proves our claim. Thus if $k$ is the number of nonisomorphic indecomposable summands of $R_{R}$, then every ascending (or descending) chain of local right ideals of $R$ contains at most $k$ terms.

Assume that $J^{k+1} \neq 0$. Choose $x_{1} \in J^{k+1}$ such that $x_{1} R$ is a local right ideal. Now, $x_{1} \in J^{k} J$ implies $x_{1}=b_{1} \alpha_{1}+\cdots+b_{t} \alpha_{t}, b_{i} \in J^{k}$ and $\alpha_{i} \in J$. Since $R$ is a dcf-ring

$$
b_{1} R+\cdots+b_{t} R=A_{1} \oplus \cdots \oplus A_{m}
$$

where each $A_{i}$ is a local right ideal contained in $J^{k}$. Then

$$
x_{1}=a_{1} \beta_{1}+\cdots+a_{m} \beta_{m}
$$

where $a_{i} \in A_{i}$ and $\beta_{i} \in J$. Let $a_{j} \beta_{j} \neq 0$. Then the mapping $x_{1} r \rightarrow a_{j} \beta_{j} r$ is an epimorphism from $x_{1} R$ onto $a_{j} \beta_{j} R$. Since $R$ is semihereditary, the epimorphism splits and as $x_{1} R$ is indecomposable we get $x_{1} R \simeq a_{j} \beta_{j} R$. Hence $x_{1} R$ is embedded properly in $A_{j}$. Let $A_{j}=x_{2} R$. Repeating the process we can find a local right ideal $x_{3} R \subset J^{k-1}$ such that $x_{2} R$ is embedded properly in $x_{3} R$. Continuing, we get a strictly ascending chain of local right ideals with $k+1$ terms, a contradiction. Hence $J^{k+1}=0$ and $R$ is semiprimary. Thus (i) implies (ii).

Conversely, let $R$ be semiprimary right semihereditary. Obviously $e R e$ is a division ring for any indecomposable idempotent $e$ of $R$. Since every finitely generated projective module over a semiperfect ring is $\mathrm{d}$-continuous by Theorem 2.1 , we get $R$ is a right dcf-ring. 
THEOREM 3.2. The following are equivalent for a ring $R$ with $J$ nil:

(i) $R$ is a right dc-ring such that $e$ Re is a division ring for every indecomposable idempotent $e$ of $R$.

(ii) $R$ is a semiprimary right hereditary ring.

Proof. Assume (i). By the above theorem, $R$ is semiprimary and every local right ideal is projective. Let $A$ be a right ideal of $R$. Since $R$ is semiprimary, $\operatorname{Rad} A$ is small in $A$. Hence $A=\Sigma_{i \in I} \oplus A_{i}$ where each $A_{i}$ is a local right ideal. Thus $A$ is projective. Hence $R$ is right hereditary and (ii) follows.

The converse is on similar lines as in Theorem 3.1.

LEMMA 3.3. Let $R$ be a right dcf-ring with $J$ nil. If $e$ is an indecomposable idempotent of $R$, then either $(e J e)^{2}=0$ or eRe is a right valuation ring.

Proof. The result is obvious if $e R e$ is a division ring. Let $e J e \neq 0$. Assume that $e R e$ is not a right valuation ring. Then there exist $a, b \in e R e$ such that $a e R e$ and $b e R e$ are not comparable. Consequently $a e R$ and $b e R$ are not comparable. Then $a e R \cap b e R=0$ by Lemma 2.7. Let $A=r(a) \cap e R$ and $B=r(b) \cap e R$. Since $A$ and $B$ are small submodules of $e R$ and $e R / A \oplus e R / B$ is d-continuous, $e R / A \simeq$ $e R / B$ by Lemma 2.6. Hence $e R / A$ is quasi-projective. It follows by Wu and Jans (1967) that $e \operatorname{Re} A=A$. Similarly $e \operatorname{Re} B=B$. Thus $e R / A \simeq e R / B$ implies that $A=B$. Let exe be a nonzero element in eJe. There exist a nonnegative integer $k$ such that $a(\text { exe })^{k} \neq 0$ and $a(\text { exe })^{k+1}=0$. Now

$$
a(e x e)^{k} e R \cap b e R \subset a e R \cap b e R=0 .
$$

Then, as proved above,

$$
r\left(a(\text { exe })^{k}\right) \cap e R=B=A .
$$

Therefore $a($ exe $)=0$. Hence eJe $\subset r(a)$. So that aeRe is a minimal right ideal in the ring $e$ Re.

Let $S$ be the right socle of $e R e$. We have proved that $S$ contains more than one minimal right ideal and $e R e / S$ is a right valuation ring. We claim that $S=e J e$. On the contrary, let $c \in e J e-S$ and let $C=r(c)$ in $e R e$. If possible, assume that $S \subset C$. As ceRe $\simeq e R e / C$, the family of all right subideals of $c e R e$ is lineraly ordered by inclusion. However, this is a contradiction since $S \subset$ ceRe and $S$ is not a minimal right ideal. Therefore $S \not \subset C$, and hence $C \subset S$. For any $b \in e R e-S$, $b R \underset{\not}{\supset} S$. So that $b e R e / C$ is not simple. Hence $\operatorname{Soc}\left(e R e^{\neq} / C\right)=S / C$. Now

$$
S=\operatorname{Soc}(c e R e) \simeq \operatorname{Soc}(e R e / C)=S / C .
$$

Thus $c S=S$. As $c$ is nilpotent, we get $S=0$, a contradiction. Hence $S=e J e$ and therefore $(e J e)^{2}=0$. This completes the proof. 
THEOREM 3.4. Let $R$ be a local ring with $J$ nil. Then $R$ is a right dcf-ring if and only if

(i) $J^{2}=0$, or

(ii) $R$ is a right valuation ring.

Proof. Necessity follows by the above lemma. Conversely, it is obvious that any local ring with $J^{2}=0$ is a right dcf-ring-in fact it has every proper right ideal semisimple. Assume that $R$ is of type (ii). Let $A$ be a finitely generated right ideal of $R$. Since $R$ is a right valuation ring, $A=a R$ for some element $a \in R$. By Lemma $2.8, r(a)$ is a two-sided ideal of $R$. Hence $a R$ is quasi-projective by $\mathrm{Wu}$ and Jans (1967). Since $R$ is semiperfect, $A$ is d-continuous by Theorem 2.1. This completes the proof.

COROllary 3.5. Any local right dcf-ring with $J$ nil is a right dc-ring whenever $J \neq \operatorname{Rad} J$.

Proof. If $J^{2}=0$, the result is obvious. Let $R$ be a right valuation ring with $J \neq \operatorname{Rad} J$. Let $x \in J-\operatorname{Rad} J$. As $\operatorname{Rad} J$ is a maximal submodule of $J$, we get $J=x R$. Hence $R$ is a principal right ideal ring with descending chain condition. Hence $R$ is a right dc-ring.

By Lemma 3.3 and Theorem 3.4 we have the following:

COROLlaRY 3.6. Let $R$ be a right dcf-ring with $J$ nil. If $e$ is an indecomposable idempotent of $R$, then $e R e$ is also a right dcf-ring.

Next we prove

LEMMA 3.7. Let e be an indecomposable idempotent in a right dcf-ring with $J$ nil. If $e R$ is not an ideal, then $e R e$ is a division ring.

PRoOF. If $e R$ is not an ideal, then there exists $x \in R$ such that $x e R \not \subset e R$. Since $x e R$ is indecomposable, $e R \not \subset x e R$. Then $x e R \cap e R=0$ by Lemma 2.7. Let $0 \neq$ eye $\in e R e$. Then $x e R \cap$ eyeR $=0$.

$$
x e R \cap \text { eyeR } \subset x e R \cap e R=0 .
$$

It follows by Proposition 2.6 that

$$
e y e R \simeq x e R \simeq e R .
$$

This implies that eye is not nilpotent. Hence eye $\notin e J e$. Therefore eJe $=0$, completing the proof. 
The proof of the following lemma is straightforward.

LEMMA 3.8. Let $R$ be a finite direct sum of rings $R_{i}$, then $R$ is a right dc-ring (or $d c$-ring) if and only if each $R_{i}$ is.

THEOREM 3.9. Let $R$ be a right dcf-ring with $J$ nil. Then $R \simeq\left(\begin{array}{ll}S & M \\ 0 & T\end{array}\right)$ where

(i) $S$ is a finite direct sum of local rings each of which has square of its radical zero or is a right valuation ring.

(ii) $T$ is a semiprimary right semihereditary ring.

(iii) $M$ is an $(S, T)$-bimodule such that every finitely generated $T$-submodule of $M$ is projective.

Proof. We can write

$$
R=e_{1} R \oplus \cdots \oplus e_{k} R \oplus f_{1} R \oplus \cdots \oplus f_{t} R
$$

where $e_{i}$ and $f_{j}$ are orthogonal indecomposable idempotents such that $e_{i} R e_{i}$ is not a division ring and $f_{j} R f_{j}$ is a division ring. By Lemma 3.7, each $e_{i} R$ is an ideal. Let $e=e_{1}+\cdots+e_{k}$. Then $1-e=f_{1}+\cdots+f_{t}$, and $R=e R e \oplus e R(1-e) \oplus$ $(1-e) R(1-e)$.

Let $S=e R e$. Then $S=e_{1} R e_{1} \oplus \cdots \oplus e_{k} R e_{k}$, and $S$ is of type (i) by Corollary 3.6 and Theorem 3.4.

Let $T=(1-e) R(1-e)=(1-e) R$. It is obvious that each right ideal of the ring $T$ is a right ideal of $R$. Hence $T$ is a right dcf-ring. Also $g T g$ is a division ring for every indecomposable idempotent $g$ of $T$. Hence $T$ is a semiprimary right semihereditary by Theorem 3.1.

Let $M=e R(1-e)$. Consider any finitely generated $T$-submodule $A$ of $M$. Then $A=\sum_{i=1}^{m} e x_{i}(1-e) R$. Since $A_{R}$ is d-continuous, $A=\sum \oplus A_{i}$ for some local $R$-modules $A_{i}$. Clearly each $A_{i}$ is a homomorphic image of $(1-e) R$, and since $A_{i} \oplus(1-e) R$ is d-continuous, the epimorphism splits. Hence $A_{R}$ is projective, and therefore $A_{T}$ is projective.

Clearly $R \simeq\left(\begin{array}{cc}S & M \\ 0 & T\end{array}\right)$. This proves the theorem.

RemarKs. (1) The converse of the above theorem is not true. Let $F[x]$ be the ring of polynomials over a field $F$, with $x^{3}=0$. Let

$$
R=\left(\begin{array}{cc}
F[x] & \left(x^{2}\right) \\
0 & F
\end{array}\right) .
$$

Then $R$ satisfies the conditions mentioned in the above theorem.

Let

$$
A=\left(\begin{array}{cc}
(x) & 0 \\
0 & 0
\end{array}\right) \text { and } B=\left(\begin{array}{cc}
0 & \left(x^{2}\right) \\
0 & 0
\end{array}\right)
$$


Then $A$ and $B$ are right ideals of $R$ with $A \cap B=0$. We have right $R$-epimorphism $f: A \rightarrow B$ defined by $f(x)=x^{2}$. This $f$ does not split. Hence $A \oplus B$ is not d-continuous by Lemma 2.4. Thus $R$ is not a right def-ring.

(2) In general $M \neq 0$. To see this let

$$
R=\left(\begin{array}{cc}
F[x] & (x) \\
0 & F
\end{array}\right)
$$

where $F[x]$ is the ring of polynomials over a field $F$, with $x^{2}=0$. This ring is a right dc-ring with $S=F[x], T=F$ and $M=(x) \neq 0$.

(3) $M=0$ whenever, in addition, $R$ is right continuous or $R$ is a left dcf-ring.

\section{Acknowledgement}

This research is partially supported by the Kuwait University research grant No. SM15. The author is extremely thankful to Professor Surjeet Singh for his valuable suggestions.

\section{References}

C. Faith (1976), Algebra. II. Ring theory (Springer-Verlag, Berlin and New York).

S. C. Goel and S. K. Jain (1976), 'Semiperfect rings with quasi-projective left ideals', Math. $J$. Okayama Univ. 19, 39-43.

S. K. Jain and S. Singh (1975), 'Rings with quasi-projective left ideals', Pacific J. Math. 60, 169-181.

A. Koehler (1971), 'Quasi-projective and quasi-injective modules', Pacific J. Math. 36, 713-720.

Y. Miyashita (1966), 'Quasi-projective modules, perfect modules and a theorem for modular lattices'. J. Fac. Sci. Hokkaido Univ. 19, 86-110.

S. Mohamed and S. Singh (1977), 'Generalization of decomposition theorems known over perfect rings', J. A ustral. Math. Soc. Ser. A 24, 496-510.

S. Mohamed and B. J. Müller (1977), Decomposition of dual contimuous modules, Lecture Notes in Math. 700, pp. 87--94. (Springer-Verlag, Berlin and New York).

F. A. Reda (1978), On continuous and dual continuous modules (M.Sc. Thesis, Kuwait University).

L. E. T. Wu and J. P. Jans (1967), 'On quasi-projective modules', Illinois J. Math. 11, 439-448.

\section{Department of Mathematics}

Kuwait University

Kuwait 